Dansk Ultralyddiagnostisk Selskab

\title{
Point-of-Care Ultrasound. A general practice perspective
}

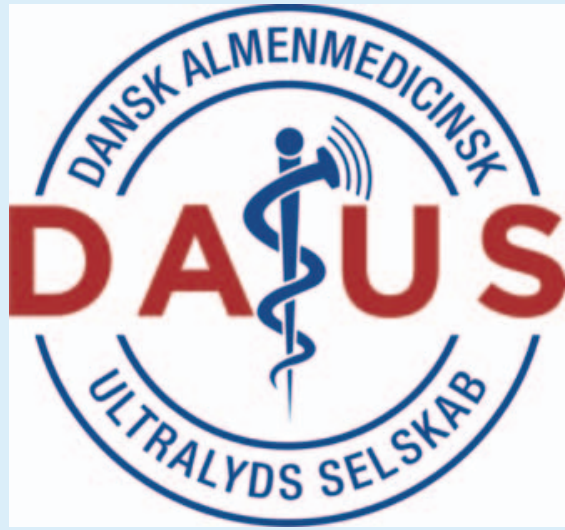

Ole Graumann (MD, PhD, Associate professor, Chairman elect DUDS)

Troels Mengel-Jørgensen, Thomas Løkkegaard, Søren Kæseler Andersen, Ulrike Mehnert, Christian Valetiner-Branth, Lars Bredahl Riisgaard, Bo Stork, Torsten Rudbæk, Christian Stjernebjerg and Nicloai Soll (all MD, GP, board members, DAUS)

Camilla Aakjær Andersen, MD, Post.Doc., and Martin Bach Jensen, Professor, PhD, GP, both Center for General Practice at Aalborg University, Aalborg, Denmark

The use of point-of-care ultrasound (POCUS) in general practice is increasing. This development may be driven by access to suitable and affordable machines together with a need for additional tools to diagnose and treat increasingly complex patients in primary care. The implementation of POCUS in general practice varies in different European countries [1, 2]. This reflects both the diversity in health care organization, specialist training, and culture among the different countries. Hence, establishing uniform guidelines and recommendations for the use of POCUS in general practice across Europe may not be feasible. Focusing on educational aspects could indeed be more relevant.

The European Federation of Societies for UItrasound in Medicine and Biology (EFSUMB)

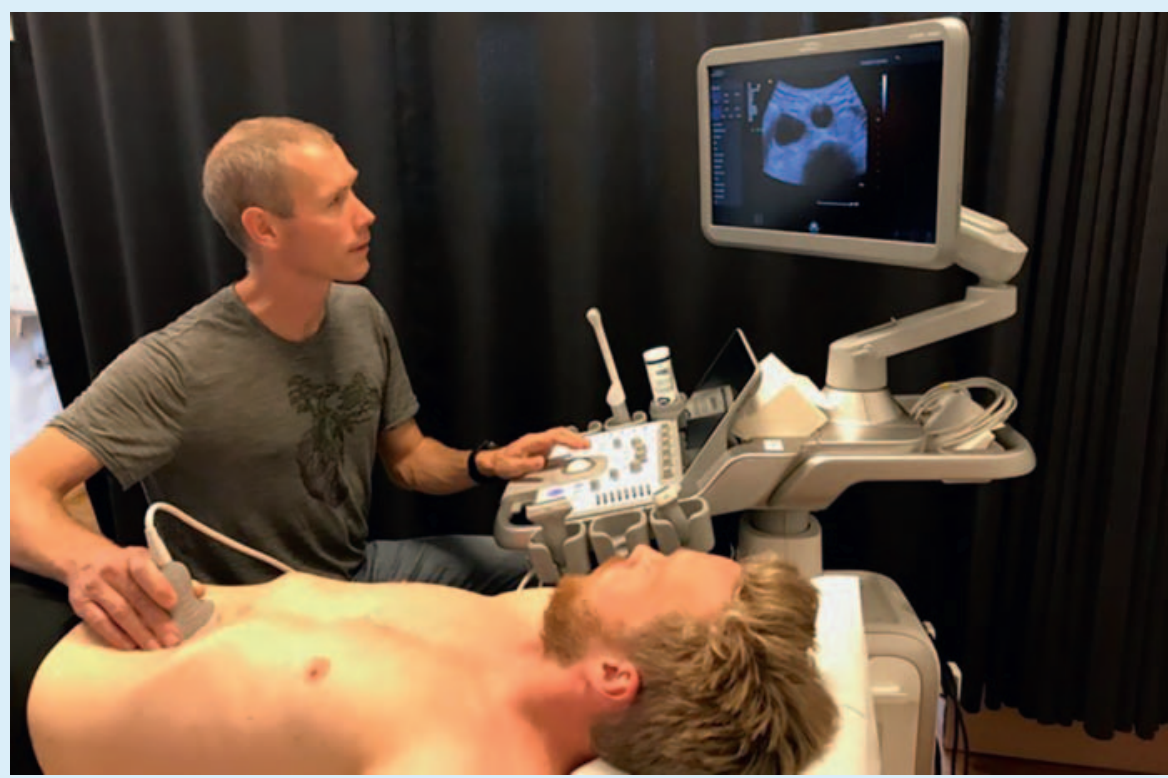

Point-of-Care Ultrasound in general practice can roll-out questions like: "Is there an abdominal aneurism: yes/no?"

has developed and published well known minimum training recommendations for the use of ultrasound in a range of medical fields. An adaptation of these training recommendations to suit the needs of general practice could help facilitate a timely and coordinated implementation process.

In Denmark, POCUS is generally used to answer selected simple clinical questions such as Does this patient have a gallstone? [3] It seems to be within reach of general practitioners to perform such focused scans with acceptable quality $[4,5]$.

Today, the use in general practice is limited to a smaller group of dedicated POCUS users. However, within a few years we expect a transition to a broad dissemination of POCUS use in the general practice community. This poses various challenges especially regarding educational capacity and quality assurance. The Danish Society for
Ultrasonography in General Practice (DAUS) have, in collaboration with researchers, set up a plan for a large-scale implementation of POCUS in Danish general practice. This includes developing training programs for general practitioners, guidelines, quality assurance programs, addressing organizational aspects, and initiation of further research. Thus, we expect the coming years to be challenging and very exciting regarding the use of POCUS in Danish general practice.

\section{References}

[1] Mengel-|ørgensen T, Jensen MB. Variation in the use of point-of-care ultrasound in general practice in various European countries. Results of a survey among experts. European Journal of General Practice 2016; 22: 274-277

[2] Andersen CA, Jensen MB, Toftegaard BS et al. Primary care physicians' access to in-house ultrasound examinations across Europe: 
a questionnaire study. BMJ Open 2019; 9: e030958

[3] Løkkegaard T, Todsen T, Nayahangan L] et al. Point-of-Care Ultrasound for General Practitioners: A Systematic Needs Assessment.
Scandinavia Journal of Primary Health Care 2020

[4] Lindgaard K, Riisgaard L. Validation of ultrasound examinations performed by general practitioners. Scand J Prim Health Care 2017; 35: $256-261$
[5] Andersen CA, Holden S, Vela J et al. Point-ofcare ultrasound in general practice: A systematic review. Annals of Family Medicine 2018; 17 : 61-69 